\title{
Tetrafurcation of the subscapular artery. Anatomical and clinical implications
}

\author{
I. Dimovelis, A. Michalinos, E. Spartalis, G. Athanasiadis, P. Skandalakis, T. Troupis \\ Department of Anatomy, National and Kapodistrian University of Athens, Greece
}

[Received: 9 August 2016; Accepted: 23 September 2016]

\begin{abstract}
Anatomic variations of axillary artery branches are commonly encountered during radiological investigation and surgical operations. Their existence can confuse interpretation of radiological results and lead to undesired complications during surgery. In this report authors describe a rare case of a subscapular arterial trunk that gave origin to thoracodorsal, circumflex scapular, posterior humeral circumflex, and lateral thoracic artery. Such a variation might cause undesired sequelae during trauma management and a variety of common flap harvesting operations including latissimus dorsi, scapular and parascapular flaps. Furthermore it presents embryological interest as it gives insight to embryologic development of axillary area. (Folia Morphol 2017; 76, 2: 312-315)
\end{abstract}

Key words: subscapular artery, posterior humeral circumflex artery, thoracodorsal artery, lateral thoracic artery, scapular flap, plastic surgery

\section{INTRODUCTION}

Arterial vascularisation of lateral thoracic wall, axilla and head of humerus derives from axillary artery (AA) branches. Variability in AA branches' origin and distribution is the rule rather than the exception. Classic pattern of 6 branches originating distinctly from AA i.e. superior thoracic artery, thoracoacromial artery, lateral thoracic artery (LTA), subscapular artery (SSA), anterior circumflex humeral artery and posterior circumflex humeral artery (PCHA) is encountered in only $27 \%$ of the cases [2]. Origin of 2 branches, irrespective of their distribution in a common trunk, is commoner than classic pattern and occurs in 37\% of the cases. Specifically common origin of SSA and LTA is encountered in $28.7 \%$ while that of SSA and PCHA occurs in $15.2 \%$ of the cases. Common origin of SSA, LTA and PCHA is a rare event and accounts for approximately $4.7 \%[2,21]$.

Textbook anatomic knowledge is often challenged by modern data and everyday surgical practice that proves variability in axillary area the rule rather than the exception $[1,2,6,12]$. In this report authors present a rare case of common origin of SSA, LTA, thoracodorsal artery (TDA) and PCHA from a common trunk. Alongside with anatomical data, we briefly review surgical implications during harvesting of arterial grafts or various flaps residing in axillary area including the subscapular flap, latissimus dorsi flap and serratus anterior flap [15].

\section{CASE REPORT}

During routine dissection of axillary region of a Caucasian cadaver for purpose of undergraduate anatomy lesson a rare anatomic variation was encountered. At the anatomical position of SSA a common trunk was encountered that gave origin to cirumflex scapular artery (CSA), TDA, LTA and PCHA (Fig. 1). This common trunk originated from AA. No other branches substituting vascularisation at corresponding areas were encountered. No other

Address for correspondence: T.G. Troupis, MD, PhD, Department of Anatomy, National and Kapodistrian University of Athens, 75 Mikras Asias str., 11527 Athens, Greece, tel: +30-210-7462388, fax: +30-210-7462398, e-mail: ttroupis@gmail.com; email: ttroupis@med.uoa.gr 


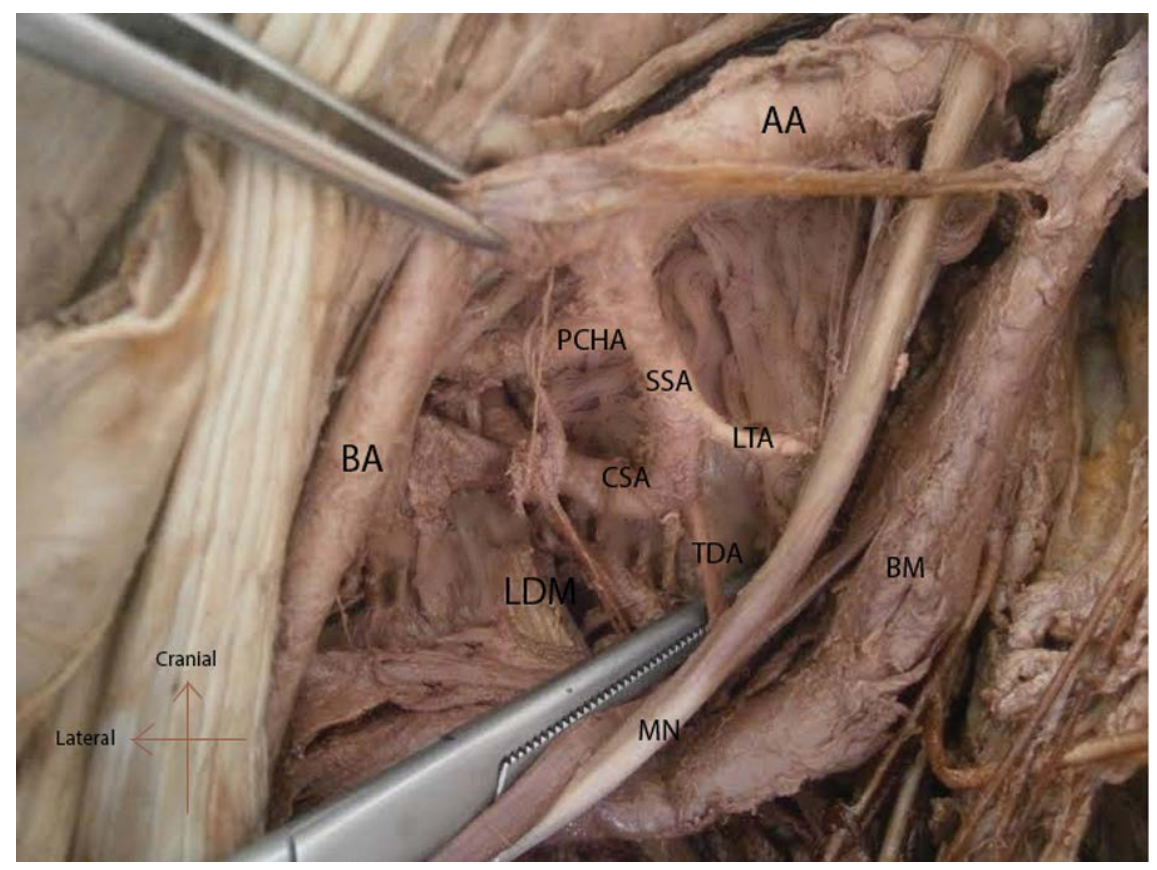

Figure 1. A common arterial trunk tetrafurcating into posterior circumflex humeral artery (PCHA), circumflex scapular artery (CSA), thoracodorsal artery (TDA) and lateral thoracic artery (LTA); SSA — subscapular artery; AA — axillary artery; BA — brachial artery; $\mathrm{BM}$ — biceps muscle; LDM — latissiumus dorsi muscle; MN — median nerve.

variations concerning upper limb were found during dissection.

The CSA followed its usual course. The length of the artery was measured at $3.43 \mathrm{~cm}$ until the dorsum of the scapula and its diameter was $0.84 \mathrm{~cm}$. LTA was distributed to the serratus anterior muscle in the extent of the fourth rib and the third and fourth intercostal spaces. Its length between its origin and insertion at serratus anterior was $8.45 \mathrm{~cm}$ and its diameter $0.48 \mathrm{~cm}$. TDA followed the inner surface and lateral border of the latissimus dorsi muscle, providing the arterial supply of the muscle during its course, and terminated about the level of the sixth rib by supplying a number of branches to the serratus anterior and the deep surface of the latissimus dorsi. The length of the TDA was $6.67 \mathrm{~cm}$ and its diameter was $0.9 \mathrm{~cm}$. The PCHA arose from the SSA and followed a dorsal course under the head of the humerus, passing alongside with the axillary nerve through the quadrilateral space. The length of the artery was approximately $3.8 \mathrm{~cm}$ and the diameter approximately $0.7 \mathrm{~cm}$.

\section{DISCUSSION}

Arterial anatomy of axillary region is complicated due to high variability in origin and course of $A A$ branches. Classic pattern of AA providing 6 separate arterial branches is present in only $27 \%$ of the cases. LTA, SSA and PCHA exhibit highest level of variability in their branching pattern and origin but relative stability is their course and area of distribution [7] Olinger and Benninger [11] studied branching pattern of LTA, SSA and PCHA and proposed their definition with respect to their course and distribution rather than their origin. Characterisation of axillary arterial network is further implicated by significant overlapping of arteries' areas of distribution. Medium sized atypical branches are often present substituting or even replacing typical branches $[7,12]$.

Similar cases to ours have been reported by Saeed et al. [14], Loukas et al. [7] and Olinger and Benninger [11]. According to Loukas et al. [7] LTA origin from SSA accounts for only $4 \%$ of the cases. Olinger and Benninger [11] calculated incidence of LTA arising from SSA at $4.2 \%$ and PCHA arising from SSA at $12 \%$. Saeed and Rufai [14] published a bilateral case of a common SSA, LTA and PCHA arterial trunk and calculated its incidence at $1.9 \%$. Their case is different than ours in common trunk's origin: theirs originated from second part of AA while ours from third part.

No complete embryological theory explains development of axillary arterial network and subsequently 
anatomical variability. Embryologic model of Singer [16] does not extend to AA branches. Rodriguez-Baeza et al. [13] based on anatomic and embryologic observation on human foetuses proposed a different model on development of axillary arterial network: According to them, morphology of arterial network does not originate from pre-existing primitive arterial networks through sprouting or obliteration but originates from capillary micro-networks in a proximal-to-distal fashion and following development of muscular and osseous elements. Hypothetically, that could explain discrepancy between high variability in origin of $A A$ branches on one side and stability in their course and area of distribution on the other side: Constancy in muscular and osseous elements of axillary area dictates constancy of terminal part of the vessels while their origin is subjected to minor environmental or genetic changes early in embryogenesis or is affected by development of close major anatomic elements, e.g. the brachial plexus

Precise knowledge of anatomy of AA branches is important in many clinical scenarios. TDA defines the posterior boundary of axillary lymph node dissection while LTA serves as a landmark for identification of long thoracic nerve [19]. Anatomic variations are a well-known and established reason for misinterpretations in surgical field and subsequent surgical errors, compromising oncologic result of various operations, especially lymph node dissections. In our case LTA, as it originated from a common trunk with SSA, entered its normal course parallel to serraturs anterior muscle at a wider angle and a lower level than usually. This could cause confusion in defining borders of complete axillary dissection, compromising oncologic result. According to Loukas et al. [7] contribution of LTA at haematosis of nipple-areola complex might be more important than initially considered and thus some nipple-areola complex necrosis might be attributed to improper ligation or destruction of this vessel.

The head of the humerus is vascularised mainly from anterior humeral circumflex artery. In case of disruption of the arterial supply such as in a fracture or trauma, the vascularisation of the head fragments during treatment can be substituted by the anastomotic vascular network of the PCHA. Considering stability in existence of these two arteries, anatomic variability does not seem important in predicting humeral head necrosis in comparison to other factors; still it should be taken in consideration in unusual clinical scenarios. Moreau et al. [10] reported an unusual case of SSA aneurysm rupture and embolisation. Rich anastomotic network of the area prevented ischaemia of head of the humerus or any other anatomic element.

Subscapular arterial system serves as a donor for arterial grafts during upper limb circulation restoration $[8,20]$.

Subscapular arterial system is widely used for creation of a large variety of flaps including the subscapular flap (vascularised from CSA), the serratus anterior flap (vascularised from TDA) and latissimus dorsi flap (vascularised from TDA). These flaps are used for coverage of medium and large size defects and combine cutaneous, subcutaneous, muscular and even bony elements $[9,18]$. Their bulkiness and wide angle of rotation make them suitable for coverage of lateral thoracic wall defects, mastectomy restoration and oromandibular defects $[7,15,17]$. Anatomic research has proven that branches of subscapular arterial tree have a significantly large and stable diameter (4-6 mm for SSA and 3-5 mm for TDA across various studies $[3,4,20]$ ) rendering them also appropriate for free flap transfer. During harvesting of those flaps the most sensitive and dangerous part of the operation is isolation of their vascular pedicle. Profound knowledge of the anatomy and readiness for existence of anatomical variations can protect the flap from improper dissection, thus preventing postoperative complications or failure of the flap. A technical issue of importance is length gaining of the vascular pedicle through ligation of its unnecessary branches. Considering small size and anatomic variations of regional branches, decision of which branches are "unnecessary" can be difficult. A significant weapon in a surgeon's armentarium for taking necessary decisions is knowledge of origin of arterial branches in the area alongside with their course, distribution and anastomotic networks.

Axillary artery branches sometime appear in uncommon clinical scenarios: Lee et al. [5] reported an unusual cirrhotic patient with spontaneous rupture of LTA. Despite continuous attempts of embolisation, haemorrhage could not be controlled.

\section{CONCLUSIONS}

Anatomical research of AA branches is of particular importance due to wide anatomic variability in the area and the large number of surgical applications. Correct identification of arterial branches is necessary 
for commonly performed general surgery operations like axillary lymph node dissection for breast cancer or trunk melanoma. Also dissection in axillary area is necessary for creation of arteriovenous fistulas for dialysis.

Furthermore, axillary area serves as a donor area for a variety of flaps used in plastic surgery. During flap harvesting, correct dissection of arterial pedicles defines immediate and long-term postoperative results. Wrong identification of an arterial stem could lead to intraoperative haemorrhage or creation of an non-well vascularised flap, prone to immediate post-operative (necrosis) or long term complications (dysfunctional flap, poor aesthetic results).

During procedures safe completion of dissections depends on surgical skill and profound knowledge of regional anatomy. Surgeons should know at least the commoner variations and be prepared to adapt surgical access and technique. Considering rarer and unpredictable variations, like the one described in this case report, no safe "road map" exists, as preoperative detailed imaging of regional arterial anatomy is rarely only performed. Yet understanding that anatomic variability is the rule rather than the exception and that rarer variations are encountered more often than anticipated might help in raising the level of suspicion thus increasing the level of safety and achieving better surgical results.

\section{REFERENCES}

1. Adachi B. Das Arteriensen Der Japaner. Kyoto: Maruzen; 1928.

2. Huelke D. Variation in the origins of the branches of the axillary artery. Anat Rec. 1959; 135(1): 33-41, doi: 10.1002/ ar.1091350105.

3. Jesus RC, Lopes MCH, Demarchi GTS, et al. The subscapular artery and the thoracodorsal branch: an anatomical study. Folia Morphol. 2008; 67(1): 58-62, indexed in Pubmed: 18335415.

4. Kawamura K, Yajima H, Kobata Y, et al. Anatomy of Yshaped configurations in the subscapular arterial system and clinical application to harvesting flow-through flaps. Plast Reconstr Surg. 2005; 116(4): 1082-1089, indexed in Pubmed: 16163099.

5. Lee TH, Park YS, Chung DJ, et al. Spontaneous rupture of the lateral thoracic artery in patients with liver cirrhosis. Korean J Intern Med. 2008; 23(3): 152-155, doi: 10.3904/ kjim.2008.23.3.152, indexed in Pubmed: 18787369.

6. Lippert H, Pabst R. Arterial variation in man. In: Arterial Variation in Man. Munich: J F Bergmann 1985: 83-84.

7. Loukas M, du Plessis M, Owens DG, et al. The lateral thoracic artery revisited. Surg Radiol Anat. 2014; 36(6): 543-549, doi: 10.1007/s00276-013-1234-x, indexed in Pubmed: 24281130.
8. Masden DL, Seruya M, Higgins JP. A systematic review of the outcomes of distal upper extremity bypass surgery with arterial and venous conduits. J Hand Surg Am. 2012; 37(11): 2362-2367, doi: 10.1016/j.jhsa.2012.07.028, indexed in Pubmed: 23044478.

9. Mayou BJ, Whitby D, Jones BM. The scapular flap: an anatomical and clinical study. Br J Plast Surg. 1982; 35(1): 8-13, indexed in Pubmed: 7066593.

10. Moreau A, Joskin J, Kreutz J, et al. Ruptured subscapular artery aneurysm and subclavian artery occlusion in a patient with type 1 neurofibromatosis: a case report. J Med Case Rep. 2014; 8: 39, doi: 10.1186/1752-1947-8-39, indexed in Pubmed: 24499535.

11. Olinger A, Benninger B. Branching patterns of the lateral thoracic, subscapular, and posterior circumflex humeral arteries and their relationship to the posterior cord of the brachial plexus. Clin Anat. 2010; 23(4): 407-412, doi: 10.1002/ca.20958, indexed in Pubmed: 20235185.

12. Paraskevas G. High or low incidence of the lateral thoracic artery's origin from the thoracoacromial artery? Surg Radiol Anat. 2015; 37(7): 887-889, doi: 10.1007/ s00276-015-1449-0.

13. Rodríguez-Baeza A, Nebot J, Ferreira B, et al. An anatomical study and ontogenetic explanation of 23 cases with variations in the main pattern of the human brachioantebrachial arteries. J Anat. 1995; 187 ( Pt 2): 473-479, indexed in Pubmed: 7592009.

14. Saeed M, Rufai AA. Median and musculocutaneous nerves: variant formation and distribution. Clin Anat. 2003; 16(5): 453-457, doi: 10.1002/ca.10096, indexed in Pubmed: 12903070.

15. Shaw RJ, Ho MW, Brown JS. Thoracodorsal artery perforator - scapular flap in oromandibular reconstruction with associated large facial skin defects. $\mathrm{Br} J$ Oral Maxillofac Surg. 2015; 53(6): 569-571, doi: 10.1016/j. bjoms.2014.10.018, indexed in Pubmed: 25857251.

16. Singer E. Embryological pattern persisting in the arteries of the arm. Anat Rec. 1933; 55(4): 403-409, doi: 10.1002/ ar.1090550407.

17. Tukiainen E. Chest wall reconstruction after oncological resections. Scand J Surg. 2013; 102(1): 9-13, doi: 10.117 7/145749691310200103, indexed in Pubmed: 23628630.

18. Uglesić V, Virag M, Varga S, et al. Reconstruction following radical maxillectomy with flaps supplied by the subscapular artery. J Craniomaxillofac Surg. 2000; 28(3): 153-160, doi: 10.1054/jcms.2000.0137, indexed in Pubmed: 10964551.

19. Ung O, Tan M, Chua B, et al. Complete axillary dissection: a technique that still has relevance in contemporary management of breast cancer. ANZ J Surg. 2006; 76(6): 518-521, doi: 10.1111/j.1445-2197.2006.03765.x, indexed in Pubmed: 16768781.

20. Valnicek SM, Mosher M, Hopkins JK, et al. The subscapular arterial tree as a source of microvascular arterial grafts. Plast Reconstr Surg. 2004; 113(7): 2001-2005, indexed in Pubmed: 15253189.

21. Xhakaza NK, Satyapal KS. Origin of the subscapular artery in the South African Black population. Folia Morphol. 2014; 73(4): 486-491, doi: 10.5603/FM.2014.0073, indexed in Pubmed: 25448908. 